\title{
Generalized Stampacchia Vector Variational-Like Inequalities and Vector Optimization Problems Involving Set-Valued Maps
}

\author{
Yanfei Chai, Sanyang Liu, and Guotao Wang \\ School of Mathematics and Statistics, Xidian University, Xian 710071, China \\ Correspondence should be addressed to Yanfei Chai; chyf_0923@163.com
}

Received 22 June 2014; Accepted 10 August 2014; Published 27 August 2014

Academic Editor: S. D. Purohit

Copyright (C) 2014 Yanfei Chai et al. This is an open access article distributed under the Creative Commons Attribution License, which permits unrestricted use, distribution, and reproduction in any medium, provided the original work is properly cited.

\begin{abstract}
We first obtain that subdifferentials of set-valued mapping from finite-dimensional spaces to finite-dimensional possess certain relaxed compactness. Then using this weak compactness, we establish gap functions for generalized Stampacchia vector variationallike inequalities which are defined by means of subdifferentials. Finally, an existence result of generalized weakly efficient solutions for vector optimization problem involving a subdifferentiable and preinvex set-valued mapping is established by exploiting the existence of a solution for the weak formulation of the generalized Stampacchia vector variational-like inequality via a Fan-KKM lemma.
\end{abstract}

\section{Introduction}

Vector variational inequality (VVI) was first introduced by Giannessi [1] in finite-dimensional spaces. Since then, much attention has been given to investigate VVIs and their generalizations. For details on VVI and their generalizations, we refer to [2-4] and the references therein. The vector variational-like inequality (VVLI), a generalization of VVI, was studied in [5-9] with applications in vector optimization problem (VOP). In [7], the equivalences among Minty VVLI, Stampacchia VVLI, both for $\eta$-subdifferentiable functions (see [10]) and for nondifferentiable nonconvex VOP were established and an existence theorem for the so-called generalized weakly efficient solutions of nondifferentiable nonconvex VOP was obtained through the relationship between VVLI and VOP. The VVLI approach was also used in $[9,11]$ to prove some existence theorems of generalized efficient solutions for nondifferentiable invex VOP. Particularly, VVLI with set-valued mapping was also considered in [12-14].

Note that some results regarding gap functions for VVLI with set-valued mappings have appeared in the literature $[12,14,15]$, but so far to the best of our knowledge there is no result available in the literature about a gap function for a generalized Stampacchia vector variational-like inequality
(GVVLI) which is defined by means of generalized subdifferentials of set-valued mapping (see Definition 3). In this paper, we introduce gap functions for our GVVLI.

Note also that there are some papers discussing solution relationships between set-valued optimization problems and vector variational-like inequalities. Miholca [13] and Zeng and $\mathrm{Li}$ [16] considered several kinds of generalized invexity for set-valued mappings and established some solution relationships between set-valued optimization problems and generalized vector variational-like inequalities problems $((M V V I)$ and $(V V L I S)$ in $[13,16]$, resp.), but they did not consider the existence results for their solutions.

Inspired and motivated by the works [7, 12-16], in this paper, we study GVVLI. We first establish generalized subdi erential mapping $\partial F$ of set-valued mapping $F$ from finitedimensional space into finite-dimension is an asymptotically compact, closed and convex-valued mapping, and then, we introduce gap functions for our GVVLI problems. In the final section of this paper, we discuss solution relationships between GVVLI and vector optimization problem involving set-valued mapping (VOP); then we obtain existence of solutions for GVVLI by using a Fan-KKM lemma and consequently an existence result for weak efficient solution of VOP is established. 


\section{Preliminaries and Notations}

Let $R^{n}$ be the $n$ dimensional space, where $n$ is a given positive integer. Set

$$
\begin{gathered}
R_{+}^{n}=\left\{y=\left(y_{1}, y_{2}, \ldots, y_{n}\right)^{T} \in R^{n} \mid y_{i} \geq 0, i=1,2, \ldots, n\right\}, \\
\text { int } R_{+}^{n}=\left\{y=\left(y_{1}, y_{2}, \ldots, y_{n}\right)^{T} \in R^{n} \mid y_{i}>0, i=1,2, \ldots, n\right\},
\end{gathered}
$$

where the symbol $T$ denotes the transpose. A nonempty subset $D$ of $R^{n}$ is said to be a cone if $\lambda D \subseteq D$ for all $\lambda \geq 0$; $D$ is said to be a convex cone if $D$ is a cone and $D+D \subseteq D$; $D$ is a closed cone if $D$ is a cone and closed; and $D$ is called a pointed cone if $D$ is a cone and $D \cap(-D)=\{0\}$. Clearly, $R_{+}^{n}$ is a closed, convex, and pointed cone of $R^{n}$, and int $R_{+}^{n}$ is a convex pointed cone of $R^{n}$. We consider the orderings induced by $R_{+}^{n}$ and int $R_{+}^{n}$ in the following form.

Let $y_{1}, y_{2} \in R^{n}$ :

$$
\begin{aligned}
& y_{1} \leq y_{2} \Longleftrightarrow y_{2}-y_{1} \in R_{+}^{n}, \\
& y_{1} \nless y_{2} \Longleftrightarrow y_{2}-y_{1} \notin R_{+}^{n}, \\
& y_{1}<y_{2} \Longleftrightarrow y_{2}-y_{1} \in \operatorname{int} R_{+}^{n}, \\
& y_{1} \nless y_{2} \Longleftrightarrow y_{2}-y_{1} \notin \operatorname{int} R_{+}^{n} .
\end{aligned}
$$

In the following sections, we denote $D=R_{+}^{n}$. Let $R^{m}$ be the $m$-dimensional space. Denote by $L\left(R^{m}, R^{n}\right)$ the space of all the continuous linear mappings from $R^{m}$ to $R^{n}$ and by $\langle l, x\rangle$ the value of $l \in L\left(R^{m}, R^{n}\right)$ at $x \in R^{m}$. Let $A \subset R^{m}$. For $x \in R^{m}$, denote by $d(x, A)$ the distance from $x$ to $A$; that is, $d(x, A)=\inf _{a \in A}\|x-a\|$. Let $F: X \rightarrow Y$ be a set-valued mapping. The graph, the epigraph, and the domain of $F$ are defined, respectively, by

$$
\begin{gathered}
\operatorname{gr} F=\{(x, y) \in X \times Y: y \in F(x)\}, \\
\text { epi } F=\{(x, y) \in X \times Y: y \in F(x)+D\}, \\
\operatorname{dom} F=\{x \in X: F(x) \neq \emptyset\} .
\end{gathered}
$$

Definition 1 (see $[17,18]$ ). Let $A \subset X$; the contingent cone of $A$ at $x_{0}$ is defined by

$$
\begin{gathered}
T\left(A, x_{0}\right)=\left\{u \in X: \exists t_{n} \longrightarrow 0^{+}, \exists u_{n} \longrightarrow u, \exists n_{0} \in N\right. \\
\left.\forall n \geq n_{0}, x_{0}+t_{n} u_{n} \in A\right\} .
\end{gathered}
$$

Definition 2 (see $[17,18])$. Let $x_{0} \in A$ and let a pair $\left(x_{0}, y_{0}\right) \in$ gr $F$ be given. The contingent epiderivative $D F\left(x_{0}, y_{0}\right)$ of $F$ at $\left(x_{0}, y_{0}\right)$ is the single-valued mapping from $X$ to $Y$ defined by

$$
\operatorname{epi}\left(D F\left(x_{0}, y_{0}\right)\right)=T\left(\operatorname{epi} F,\left(x_{0}, y_{0}\right)\right) \text {. }
$$

$F$ is called epidifferentiable at $\left(x_{0}, y_{0}\right)$ if the contingent epiderivative $D F\left(x_{0}, y_{0}\right)$ of $F$ at $\left(x_{0}, y_{0}\right)$ exists.

Recall that the contingent cone $T\left(\right.$ epi $\left.F,\left(x_{0}, y_{0}\right)\right)$ consists of all tangent vectors $h=\lim _{n \rightarrow+\infty} \lambda_{n}\left(\left(x_{n}, y_{n}\right)-\left(x_{0}, y_{0}\right)\right)$ with $\left(x_{0}, y_{0}\right)=\lim _{n \rightarrow+\infty}\left(x_{n}, y_{n}\right)$ and $\left(x_{n}, y_{n}\right) \in$ epi $F$ for all $n \in N$ and $\lambda_{n}>0$.
Definition 3 (see [19]). Suppose that $F$ is epidifferentiable at $\left(x_{0}, y_{0}\right) \in \operatorname{gr} F$; then

(i) a continuous linear map $l: X \rightarrow Y$, with $l(x) \leq$ $D F\left(x_{0}, y_{0}\right)(x)$, for all $x \in \operatorname{dom}\left(D F\left(x_{0}, y_{0}\right)\right)$ is called a subgradient of $F$ at $\left(x_{0}, y_{0}\right)$;

(ii) the set $\partial F\left(x_{0}, y_{0}\right)=\left\{l: X \rightarrow Y: l(x) \leq D F\left(x_{0}\right.\right.$, $\left.y_{0}\right)(x), \forall x \in \operatorname{dom}\left(\operatorname{DF}\left(x_{0}, y_{0}\right)\right\}$ of all subgradients $l$ of $F$ at $\left(x_{0}, y_{0}\right)$ is called the subdifferential of $F$ at $\left(x_{0}, y_{0}\right)$.

The following notions are based on the concept of contingent epiderivative.

Lemma 4 (see [19]). Let $X$ and $Y$ be real normed spaces, $A$ a subset of $X$, and $D$ a convex cone. $F: A \rightarrow 2^{Y},\left(x_{0}, y_{0}\right) \in$ gr $F$, and let contingent epiderivative $\operatorname{DF}\left(x_{0}, y_{0}\right)$ of $F$ at $\left(x_{0}, y_{0}\right)$ exist. Then the subdifferential $\partial F\left(x_{0}, y_{0}\right)$ is convex.

Lemma 5 (see [19]). Let assumptions of Lemma 4 be satisfied and let $D$ be closed. If all subgradients are bounded, then the subdifferential is closed in the linear space of all bounded maps.

For the spacial case, where $X=R^{m}$ and $Y=R^{n}$, linear maps are always bounded, so the subdifferential is closed whenever $D$ is closed.

Corollary 6. If $X=R^{m}, Y=R^{n}$, and $D=R_{+}^{n}$, then subdifferential of $F$ at $(x, y) \in$ gr $F$ is a closed and convex set in the linear space $L\left(R^{m}, R^{n}\right)$.

We propose the following relaxed compactness which will be needed for the following sections.

Definition 7 (see [20]). (i) Let $l_{n}$ and $l$ be in $L(X, Y)$. The sequence $\left\{l_{n}\right\}$ is said to pointwisely converge to $l$ and written as $l=p-\lim l_{n}$ if $\lim _{n \rightarrow+\infty} l_{n}(x)=l(x)$ for all $x \in X$.

(ii) Let $l_{n}$ and $l$ be in $L(X, Y)$. The sequence $\left\{l_{n}\right\}$ is said to asymptotically pointwisely converge to $l$ if

(a) sequence $\left\{l_{n}\right\}$ is bounded and it has a subsequence $\left\{l_{n_{k}}\right\}$ and $l \in L(X, Y)$ such that $l=p-\lim l_{n_{k}}$;

(b) sequence $\left\{l_{n}\right\}$ with $\lim \left\|l_{n}\right\|=\infty$ and the sequence $\left\{l_{n} /\left\|l_{n}\right\|\right\}$ has a subsequence which pointwisely converges to some $l \in L(X, Y) \backslash\{0\}$.

(iii) A subset $M \subset L(X, Y)$ is called asymptotically pointwisely compact or asymptotically $p$-compact if each sequence $\left\{l_{n}\right\} \subset M$ has a subsequence which asymptotically pointwisely converges to $l \in L(X, Y)$.

(iv) If, in (iii), pointwise convergence, that is, $p$-lim, is replaced by convergence, that is, lim, a subset $M \subset L(X, Y)$ is called asymptotically compact.

Remark 8. (i) If $X$ and $Y$ are finite dimensional, a convergence occurs if and only if the corresponding pointwise convergence does.

(ii) If $X$ and $Y$ are finite dimensional, every subset is asymptotically $p$-compact and asymptotically compact.

(iii) If $X$ and $Y$ are finite dimensional, then every subset of $L(X, Y)$ is asymptotically $p$-compact. 
Remark 9. By Remark 8, if assumptions of Corollary 6 are satisfied and $\partial F(x, y)$ exists at $(x, y) \in \operatorname{gr} F$, then $\partial F(x, y)$ is an asymptotically compact closed and convex subset in $L\left(R^{m}, R^{n}\right)$.

Remark 10. From Remark 9, if $t \partial F(x, y) \subseteq \partial F(x, y)$ for all $t \in(0,1]$, whenever $\partial F(x, y)$ exists at $(x, y) \in \operatorname{gr} F$, then each sequence $\left\{l_{n}\right\} \subset \partial F(x, y)$ has a subsequence which asymptotically converges to some $l \in \partial F(x, y)$. In the following sections we always assume that $t \partial F(x, y) \subseteq \partial F(x, y)$ for all $t \in(0,1]$, whenever $\partial F(x, y)$ exists at $(x, y) \in \operatorname{gr} F$.

Example 11. Let $X=Y=R, A=[0,3]$, and $D=R_{+}=\{t \in$ $R: t \geq 0\}$ and let $F$ be defined by

$$
F(x)=\{y \in R \mid x \leq y \leq 2 x+1\} .
$$

Then $(0,0) \in \operatorname{gr} F$ and $\operatorname{DF}(0,0)(x)=x$ for all $x \geq$ $0(\operatorname{dom}(D F(0,0))=[0,+\infty))$; therefore, $\partial F(0,0)=\{l \in R \mid$ $-\infty<l \leq 1\}$. Obviously, $t \partial F(0,0) \subseteq \partial F(0,0)$ for all $t \in(0,1]$.

Definition 12. A set $A \subset X$ is said to be an invex set if there exists a function $\eta: X \times X \rightarrow X$ such that $\forall x, y \in A, \forall \lambda \in$ $[0,1]$, and $y+\lambda \eta(x, y) \in A$.

Remark 13. Obviously, the convex set is a particular case of the invex set if $\eta(x, y)=x-y$, but the converse does not hold; see the following example.

Example 14. Let $A=[-3,0] \cup[1,2] \subset R$ and let $\eta$ be defined by

$$
\eta(x, y)= \begin{cases}x-y, & x, y \in[-3,0] \text { or } x, y \in[1,2] \\ x, & x \in[-3,0], y \in[1,2] \\ 0, & x \in[1,2], y \in[-3,0]\end{cases}
$$

We note that $A$ is an invex set with respect to the above $\eta$.

Throughout this paper, we always assume that $A$ is an invex subset of $R^{m}$, the function $\eta$ is defined on $A$, that is, $\eta: A \times A \rightarrow R^{m}$, and $F: A \rightarrow 2^{R^{n}}$ is a set-valued mapping with $\partial F(x, y)$ existing at every $(x, y) \in \operatorname{gr} F$ and $x \in A$. By Remark 9, we obtain $\partial F: R^{m} \times R^{n} \rightarrow 2^{L\left(R^{m}, R^{n}\right)}$, when it exists, a set-valued mapping with an asymptotically compact closed convex-valued $\partial F(x, y)$ at $(x, y) \in \operatorname{gr} F$. Furthermore, under mild assumptions, $\partial F(x, y)$ has certain compactness (see Remark 10). Next, we consider the following strong and weak generalized Stampacchia vector variational-like inequalities (GVVLI):

(SGVVLI) find $\left(x_{0}, y_{0}\right) \in \operatorname{gr} F$ and $l_{0} \in \partial F\left(x_{0}, y_{0}\right)$ such that

$$
\left\langle l_{0}, \eta\left(x, x_{0}\right)\right\rangle \nless_{\mathrm{int} D} 0, \quad \forall x \in A
$$

and

(WGVVLI) find $\left(x_{0}, y_{0}\right) \in \operatorname{gr} F$ such that $\forall x \in A$ and there exist $l_{0}(x) \in \partial F\left(x_{0}, y_{0}\right)$ satisfying

$$
\left\langle l_{0}(x), \eta\left(x, x_{0}\right)\right\rangle \nless_{\operatorname{int} D} 0 .
$$

We denote by $S_{\text {SGVVLI }}$ and $S_{\text {WGVVLI }}$ the solution sets of SGVVLI and WGVVLI, respectively. Obviously, $S_{\text {SGVVLI }} \subseteq$ $S_{\text {WGVVLI}}$.

\section{Gap Functions}

In this section, we assume that $\eta$ defined on $A$ is an open map such that $\eta(x, z)+\eta(z, x)=0$ for all $x, z \in A$. Then, we introduce the concept of gap functions for our SGVVLI and WGVVLI.

Definition 15. Let $A$ be the domain of SGVVLI (resp., WGVVLI). A function $h: A \times R^{n} \rightarrow R \cup\{+\infty\}$ is said to be a gap function for SGVVLI (resp., WGVVLI) if it satisfies the following properties:

(i) $h(x, y) \geq 0, \forall x \in A$ with $y \in F(x)$;

(ii) $h\left(x_{0}, y_{0}\right)=0$ if and only if $\left(x_{0}, y_{0}\right)$ (where $y_{0} \in F\left(x_{0}\right)$ ) solves SGVVLI (resp., WGVVLI).

Let $x \in A$ with $y \in F(x), u \in \operatorname{dom}(\operatorname{DF}(x, y))$, and $l \in$ $\partial F(x, y)$. Denote

$$
\langle l, u\rangle=\left(\langle l, u\rangle_{1}, \ldots,\langle l, u\rangle_{n}\right)
$$

that is, $\langle l, u\rangle_{i}$ is the $i$ th component of $\langle l, u\rangle, i=1,2, \ldots, n$. Now, we define $\varphi_{\partial F}: A \rightarrow R \cup\{+\infty\}$ as

$$
\begin{array}{r}
\varphi_{\partial F}(x, y)=\inf _{l \in \partial F(x, y)} \sup _{z \in A} \min _{1 \leq i \leq n}\langle l, \eta(x, z)\rangle_{i}, \\
\forall x \in A \text { with } y \in F(x),
\end{array}
$$

where $y \in F(x)$ such that $\partial F(x, y)$ exists. For $x \in A$ and $y \in F(x)$, let

$$
S_{x}^{y}=\{l \mid l: A \rightarrow \partial F(x, y)\}
$$

where $S_{x}^{y}$ is the set of all operators $l$ from $A$ to $\partial F(x, y)$. Let $x \in A,(x, y) \in \operatorname{gr} F$, and $l \in S_{x}^{y}$; then $l(z) \in \partial F(x, y), \forall z \in A$. We also define $\phi_{\partial F}: A \rightarrow R \cup\{+\infty\}$ as follows:

$$
\begin{array}{r}
\phi_{\partial F}(x, y)=\inf _{l \in S_{x}^{y}} \sup _{z \in A} \min _{1 \leq i \leq n}\langle l(z), \eta(x, z)\rangle_{i}, \\
\forall x \in A \text { with } y \in F(x) .
\end{array}
$$

Lemma 16. Let $X$ be a normed space and $\emptyset \neq M \subseteq X$ an asymptotically compact closed convex subset; then there exists $x_{0} \in M$ such that $\left\|x_{0}\right\|=\inf \{\|x\| \mid x \in M\}$.

Proof. Let $d=\inf _{x \in M}\{\|x\|\}$; then for any $n \in N$, there exists $x_{n} \in M$ such that

$$
d \leq\left\|x_{n}\right\|<d+\frac{1}{n} \leq d+1 .
$$

So sequence $\left\{x_{n}\right\}$ is bounded; by assumption $M$ being an asymptotically compact closed convex subset, there exists a subsequence $\left\{x_{n_{k}}\right\}$ of $\left\{x_{n}\right\}$ such that $x_{n_{k}} \rightarrow x_{0} \in M$. For $x_{0}$, by applying Hahn-Banach theory, there exists $f \in X^{*}$ such that $\|f\|=1, f\left(x_{0}\right)=\left\|x_{0}\right\|$. Hence, on the one hand,

$$
x_{0} \in M \Longrightarrow\left\|x_{0}\right\| \geq d
$$


on the other hand,

$$
\left\|x_{0}\right\|=f\left(x_{0}\right)=\lim _{k \rightarrow \infty} f\left(x_{n_{k}}\right) \leq \varliminf_{k \rightarrow \infty}\|f\|\left\|x_{n_{k}}\right\|=d .
$$

Therefore, $\left\|x_{0}\right\|=d=\inf \{\|x\| \mid x \in M\}$.

Theorem 17. Suppose that $\partial F(x, y) \neq \emptyset$ for all $x \in A$ with $y \in F(x)$ such that $t \partial F(x, y) \subseteq \partial F(x, y)$ for all $t \in(0,1]$. If the function $\eta$ is an open map such that $\eta(x, z)+\eta(z, x)=0$ for all $x, z \in A$. Then, the following statements are true.

(i) The function $\varphi_{\partial F}$ defined by (11) is a gap function for SGVVLI.

(ii) The function $\phi_{\partial F}$ defined by (13) is a gap function for WGVVLI.

Proof. (i) By assumptions, for each $x \in A$ with $(x, y) \in$ gr $F, \partial F(x, y)$ is an asymptotically compact closed convex set. Then, the function $\varphi_{\partial F}$ given by (11) is well defined and from Lemma 16 we have

$$
\begin{array}{r}
\varphi_{\partial F}(x, y)=\min _{l \in \partial F(x, y)} \sup _{z \in A} \min _{1 \leq i \leq n}\langle l, \eta(x, z)\rangle_{i}, \\
\forall x \in A \text { with }(x, y) \in \text { gr } F .
\end{array}
$$

It is immediate that

$$
\sup _{z \in A} \min _{1 \leq i \leq n}\langle l, \eta(x, z)\rangle_{i} \geq 0,
$$

$\forall x \in A$ with $(x, y) \in \operatorname{gr} F, \quad \forall l \in \partial F(x, y)$,

so that $\varphi_{\partial F}(x, y) \geq 0, \forall x \in A$ with $y \in F(x)$. Let $x_{0} \in A$ with $y_{0} \in F\left(x_{0}\right)$. We note that $\varphi_{\partial F}\left(x_{0}, y_{0}\right)=0$ if and only if there exists $l_{0} \in \partial F\left(x_{0}, y_{0}\right)$ such that

$$
\sup _{z \in A} \min _{1 \leq i \leq n}\left\langle l_{0}, \eta\left(x_{0}, z\right)\right\rangle_{i}=0,
$$

or

$$
\min _{1 \leq i \leq n}\left\langle l_{0}, \eta\left(x_{0}, z\right)\right\rangle_{i}=0, \quad \forall z \in A,
$$

which is equivalent to

$$
\left\langle l_{0}, \eta\left(z, x_{0}\right)\right\rangle \Varangle_{\text {int } D} 0, \quad \forall z \in A ;
$$

that is, $\left(x_{0}, y_{0}\right) \in S_{\text {SGVVLI}}$.

(ii) Since for any given $x \in A$ with $y \in F(x)$ and $l \in S_{x}^{y}$

$$
\sup _{z \in A} \min _{1 \leq i \leq n}\langle l(z), \eta(x, z)\rangle_{i} \geq \min _{1 \leq i \leq n}\langle l(x), \eta(x, x)\rangle_{i}=0,
$$

we obtain

$$
\phi_{\partial F}(x, y)=\inf _{l \in S_{x}^{y}} \sup _{z \in A} \min _{1 \leq i \leq n}\langle l(z), \eta(x, z)\rangle_{i} \geq 0,
$$

$\forall x \in A$ with $y \in F(x)$.

We assume that $\left(x_{0}, y_{0}\right) \in$ gr $F$ solves WGVVLI. Then, for any $z \in A$, there is a $l(z) \in \partial F\left(x_{0}, y_{0}\right)$ such that

$$
\left\langle l(z), \eta\left(z, x_{0}\right)\right\rangle \not_{\text {int } D} 0,
$$

which implies that

$$
\min _{1 \leq i \leq n}\left\langle l(z), \eta\left(x_{0}, z\right)\right\rangle_{i} \leq 0 .
$$

Thus an operator $l$ from $A$ to $\partial F\left(x_{0}, y_{0}\right)$ has been defined. It follows that $l \in S_{x_{0}}^{y_{0}}$ and

$$
\min _{1 \leq i \leq n}\left\langle l(z), \eta\left(x_{0}, z\right)\right\rangle_{i} \leq 0, \quad \forall z \in A
$$

Therefore,

$$
\sup _{z \in A} \min _{1 \leq i \leq n}\left\langle l(z), \eta\left(x_{0}, z\right)\right\rangle_{i} \leq 0
$$

and so

$$
\phi_{\partial F}\left(x_{0}, y_{0}\right)=\inf _{l \in S_{x_{0}}^{y_{0}}} \sup _{z \in A} \min _{1 \leq i \leq n}\left\langle l(z), \eta\left(x_{0}, z\right)\right\rangle_{i} \leq 0 .
$$

Since $\phi_{\partial F}(x, y) \geq 0$ for all $x \in A$ with $y \in F(x)$, it follows that $\phi_{\partial F}\left(x_{0}\right)=0$. Conversely, suppose that $\phi_{\partial F}\left(x_{0}\right)=0$ and $y_{0} \in F\left(x_{0}\right)$. Let

$$
\sup _{z \in A} \min _{1 \leq i \leq n}\left\langle l(z), \eta\left(x_{0}, z\right)\right\rangle_{i}, \quad \forall l \in S_{x_{0}}^{y_{0}} .
$$

Since $\phi_{\partial F}\left(x_{0}\right)=0$, for $\epsilon_{1}>\epsilon_{2}>\cdots>\epsilon_{m}>\cdots>0$ with $\epsilon_{m}=\epsilon_{1} / 2^{m-1}$, there exist $l^{m} \in S_{x_{0}}^{y_{0}}$ such that

$$
\sup _{z \in A} \min _{1 \leq i \leq n}\left\langle l^{m}(z), \eta\left(x_{0}, z\right)\right\rangle_{i} \leq \epsilon_{m},
$$

or equivalently

$$
\min _{1 \leq i \leq n}\left\langle l^{m}(z), \eta\left(x_{0}, z\right)\right\rangle_{i} \leq \epsilon_{m}, \quad \forall z \in A, \forall m \in N,
$$

which implies

$$
\min _{1 \leq i \leq n}\left\langle\frac{l^{m}(z)}{\left\|l^{m}(z)\right\|}, \eta\left(x_{0}, z\right)\right\rangle_{i} \leq \frac{\epsilon_{m}}{\left\|l^{m}(z)\right\|}, \quad \forall z \in A, \forall m \in N .
$$

Observe that $\left\{l^{m}(z)\right\} \subseteq \partial F\left(x_{0}, y_{0}\right)$ for all $z \in A$. Since $\partial F\left(x_{0}, y_{0}\right)$ is asymptotically compact closed convex set and by Remark 10, for any $z \in A,\left\{l^{m}(z)\right\}$ has an asymptotically convergent subnet with limit $l_{0}(z) \in \partial F\left(x_{0}, y_{0}\right)$. Without loss of generality, we may assume that $\left\{l^{m}(z)\right\}$ asymptotically converges to $l_{0}(z) \in \partial F\left(x_{0}, y_{0}\right)$. Consequently, an operator $l_{0}: A \rightarrow \partial F\left(x_{0}, y_{0}\right)$ has been defined; that is, $l_{0} \in S_{x_{0}}^{y_{0}}$ and

$$
\left\langle l^{m}(z), \eta\left(x_{0}, z\right)\right\rangle \longrightarrow\left\langle l_{0}(z), \eta\left(x_{0}, z\right)\right\rangle, \quad \forall z \in A,
$$

or

$$
\left\langle\frac{l^{m}(z)}{\left\|l^{m}(z)\right\|}, \eta\left(x_{0}, z\right)\right\rangle \longrightarrow\left\langle l_{0}(z), \eta\left(x_{0}, z\right)\right\rangle, \quad \forall z \in A,
$$

which imply

$$
\min _{1 \leq i \leq n}\left\langle l^{m}(z), \eta\left(x_{0}, z\right)\right\rangle_{i} \longrightarrow \min _{1 \leq i \leq n}\left\langle l_{0}(z), \eta\left(x_{0}, z\right)\right\rangle_{i},
$$


or

$$
\begin{array}{r}
\min _{1 \leq i \leq n}\left\langle\frac{l^{m}(z)}{\left\|l^{m}(z)\right\|}, \eta\left(x_{0}, z\right)\right\rangle_{i} \rightarrow \min _{1 \leq i \leq n}\left\langle l_{0}(z), \eta\left(x_{0}, z\right)\right\rangle_{i}, \\
\forall z \in A .
\end{array}
$$

Taking the limit for $m \rightarrow+\infty$ in (31) or (32), we obtain

$$
\min _{1 \leq i \leq n}\left\langle l_{0}(z), \eta\left(x_{0}, z\right)\right\rangle_{i} \leq 0, \quad \forall z \in A .
$$

Then, for any $z \in A$, there exists $l_{0}(z) \in \partial F\left(x_{0}, y_{0}\right)$ such that

$$
\min _{1 \leq i \leq n}\left\langle l_{0}(z), \eta\left(z, x_{0}\right)\right\rangle_{i} \geq 0
$$

which implies that, for any $z \in A$, there exists $l_{0}(z) \in$ $\partial F\left(x_{0}, y_{0}\right)$ such that

$$
\left\langle l_{0}(z), \eta\left(z, x_{0}\right)\right\rangle \not_{\mathrm{int} D} 0 ;
$$

that is, $\left(x_{0}, y_{0}\right) \in S_{\mathrm{WGVVLI}}$. This completes the proof.

Example 18. Let $X=Y=R, A=[0,5]$, and $D=R_{+}=\{t \in$ $R: t \geq 0\}$ and let $F$ and $\eta$ be defined by

$$
\begin{aligned}
& F(x)=\{y \in R \mid 0 \leq y \leq-x+5\}, \\
& \eta(x, y)=\frac{1}{2}(x-y), \quad \forall x, y \in A .
\end{aligned}
$$

Then, $\eta(x, y)+\eta(y, x)=0$ for all $x, y \in A$. Since $(0,0) \in \operatorname{gr} F$ and

$$
T(\text { epi } F,(0,0))=\{(x, y) \mid x \geq 0, y \geq 0\}
$$

therefore, we get

$$
\operatorname{DF}(0,0)(x)=\{0\},
$$

when $x \in \operatorname{dom}(D F(0,0))$, and, consequently,

$$
\partial F(0,0)=\{l \in R \mid-\infty<l \leq 0\} .
$$

So, $t \partial F(0,0) \subseteq \partial F(0,0)$ hold for all $t \in(0,1]$.

\section{Existence Result for Vector Optimization Problems}

Vector variational inequalities (or their generalized form) have been shown to be a useful tool in vector optimization. Some authors have proved the equivalence between them; see $[8,13,16]$. In this section, we prove the existence of generalized weakly efficient solutions for the following invex vector optimization problem (VOP) through the relationship between GVVLI and VOP by using a Fan-KKM lemma:

$$
\operatorname{Min}\{F(x): x \in A\},
$$

where $F$ is a set-valued mapping as above and $A$ is an invex set.

We denote set-valued optimization problems (44) as (SOP). Let $x_{0} \in A, y_{0} \in F\left(x_{0}\right)$.
Definition 19. A pair $\left(x_{0}, y_{0}\right) \in$ gr $F$ is called weak efficient solution of $F$ on $A$ if $\left(F(A)-y_{0}\right) \bigcap(-$ int $D)=\emptyset$.

The set of all weak efficient solutions of (SOP) is denoted by $W \operatorname{Min}(F, A)$.

Definition 20. Let $F: A \rightarrow 2^{Y}$ be a set-valued mapping, $x_{0} \in A$, and $\left(x_{0}, y_{0}\right) \in$ gr $F . F$ is called strict pseudoinvex with respect to $\eta: A \times A \rightarrow X$ at $\left(x_{0}, y_{0}\right)$ if $\forall x \in A, \forall y \in F(x)$, and $\forall l \in \partial F\left(x_{0}, y_{0}\right), y-y_{0} \notin \operatorname{int} D \Rightarrow \ln \left(x, x_{0}\right) \notin \operatorname{int} D$.

Remark 21. We note that $F$ of Example 18 is strict pseudoinvex with respect to $\eta$ at $(0,0)$. In fact, since $0 \in F(x)$ for all $x \in$ $A$ and taking any $l \in \partial F(0,0)$, we have $0-0=0 \notin$ int $R_{+}$and obviously $\operatorname{l\eta }(u, 0)=(1 / 2) l(u-0)=(1 / 2) l(u) \leq 0 \notin$ int $R_{+}$, where $u \in \operatorname{dom}(\operatorname{DF}(0,0))$ and $u \geq 0$.

Definition 22. Let $F: A \rightarrow 2^{Y}$ be a set-valued mapping. $F$ is said to be $D$-preinvex with respect to $\eta$ on $A$ if $\forall x, z \in A$, $\forall \lambda \in[0,1], \lambda F(x)+(1-\lambda) F(z) \subset F(z+\lambda \eta(x, z))+D$.

Definition 23. $F$ is said to be upper semicontinuous at $\bar{x} \in A$ if for any neighbourhood $V$ of the origin in $Y$ there exists a neighbourhood $U$ of the origin in $X$ such that

$$
F(x) \subseteq F(\bar{x})+V, \quad \forall x \in(\bar{x}+U) \cap A .
$$

Lemma 24 (Lemma 1.1, [21]). Let a multifunction $F: X \rightarrow$ $2^{Y}$. If $F$ is compact-valued multifunction, then $F$ is upper semicontinuous if and only if for every net $\left\{\left(x_{n}, y_{n}\right)\right\}$ in $\operatorname{gr} F$ that satisfies $x_{n} \rightarrow x$ for some $x \in X$ the net $\left\{y_{n}\right\}$ has a subnet converging to a point in $F(x)$.

The following lemma will give a similar but more generalized result.

Lemma 25. Let a multifunction $F: X \rightarrow 2^{Y}$. If $F$ is asymptotically closed compact-valued multifunction and upper semicontinuous such that $t F(x) \subseteq F(x)$ for all $t \in(0,1]$ and $x \in X$, then for every net $\left\{\left(x_{n}, y_{n}\right)\right\}$ in gr $F$ that satisfies $x_{n} \rightarrow$ $x$ for some $x \in X$ the net $\left\{y_{n}\right\}$ has a subnet asymptotically converging to a point in $F(x)$.

Proof. Take a system $\left\{V_{\mu}\right\}, \mu \in I$ of neighbourhoods of the origin in $Y$ such that $\left\{V_{\mu}\right\} \rightarrow\{0\}$, where $\left\{V_{\mu}\right\} \rightarrow\{0\}$ stands for $V_{\nu} \subseteq V_{\mu}, \nu \geq \mu$, and $\bigcap_{\mu \in I} V_{\mu}=\{0\}$ and $I$ is an ordered index set. From the upper semicontinuity of $F$ and $x_{n} \rightarrow x$, one claims that for any $V_{\mu}$ there exists $n_{0}=n_{0}(\mu)$ such that $F\left(x_{n}\right) \subseteq F(x)+V_{\mu}$ for all $n \geq n_{0}$. Consequently, $y_{n} \in F\left(x_{n}\right)$ can be written as $y_{n}=\bar{y}_{n}+v_{\mu}$ with $\bar{y}_{n} \in F(x)$ and $\nu_{\mu} \in V_{\mu}$. From the asymptotic compactness of $F(x)$ and $\left\{V_{\mu}\right\} \rightarrow\{0\}$, without loss of generality, we may assume that $\left\{\bar{y}_{n}\right\}$ asymptotically converges to $y \in Y$. Furthermore, since $t F(x) \subseteq F(x)$ for all $t \in(0,1]$ and the closedness assumption of $F(x)$, we can conclude that $y \in F(x)$. This implies that $\left\{y_{n}\right\}$ asymptotically converges to $y \in F(x)$. The proof is complete.

Proposition 26. Let $A$ be an invex set with respect to $\eta$ and let $F: A \rightarrow 2^{Y}$ be D-preinvex with respect to the same $\eta$ 
on $A$. Let $x_{0} \in A,\left(x_{0}, y_{0}\right) \in \operatorname{gr} F$, and $\partial F\left(x_{0}, y_{0}\right) \neq \emptyset$. Then $\forall l \in \partial F\left(x_{0}, y_{0}\right), \forall x \in A, y \in F(x)$, and

$$
y-y_{0} \in\left\langle l, \eta\left(x, x_{0}\right)\right\rangle+D .
$$

Proof. Taking arbitrary elements $x \in A$ and $y \in F(x)$, for all $n \in N$, we define a sequence $\left(x_{n}, y_{n}\right)_{n \in N}$ with

$$
\begin{aligned}
& x_{n}=x_{0}+\frac{1}{n} \eta\left(x, x_{0}\right), \\
& y_{n}=y_{0}+\frac{1}{n}\left(y-y_{0}\right) .
\end{aligned}
$$

Since $A$ is an invex set and $F$ is a $D$-preinvex map, it follows for all $n \in N$

$$
\begin{gathered}
x_{n}=x_{0}+\frac{1}{n} \eta\left(x, x_{0}\right) \in A \\
y_{n}=\left(1-\frac{1}{n}\right) y_{0}+\frac{1}{n} y \in F\left(x_{0}+\frac{1}{n} \eta\left(x, x_{0}\right)\right) \\
+D=F\left(x_{n}\right)+D .
\end{gathered}
$$

So, $\left(x_{n}, y_{n}\right)_{n \in N}$ is a sequence in the epigraph of $F$ converging to $\left(x_{0}, y_{0}\right)$. Moreover we obtain

$$
\lim _{n \rightarrow \infty} n\left(x_{n}-x_{0}, y_{n}-y_{0}\right)=\left(\eta\left(x, x_{0}\right), y-y_{0}\right) .
$$

Consequently, we get

$$
\left(\eta\left(x, x_{0}\right), y-y_{0}\right) \in T\left(\text { epi } F,\left(x_{0}, y_{0}\right)\right)=\operatorname{epi}\left(D F\left(x_{0}, y_{0}\right)\right) \text {, }
$$

implying that

$$
y-y_{0} \in \operatorname{DF}\left(x_{0}, y_{0}\right)\left(\eta\left(x, x_{0}\right)\right)+D
$$

It is clear that

$$
\forall x \in A, \quad \eta\left(x, x_{0}\right) \in \operatorname{dom} \operatorname{DF}\left(x_{0}, y_{0}\right) .
$$

By the definition of the subdifferential $\partial F\left(x_{0}, y_{0}\right)$, for all $l \epsilon$ $\partial F\left(x_{0}, y_{0}\right)$, we have

$$
\begin{array}{r}
\left\langle l, \eta\left(x, x_{0}\right)\right\rangle \leq D F\left(x_{0}, y_{0}\right)\left(\eta\left(x, x_{0}\right)\right), \\
\forall \eta\left(x, x_{0}\right) \in \operatorname{dom} D F\left(x_{0}, y_{0}\right) ;
\end{array}
$$

that is,

$$
D F\left(x_{0}, y_{0}\right) \eta\left(x, x_{0}\right) \in\left\langle l, \eta\left(x, x_{0}\right)\right\rangle+D .
$$

Considering (51) and (54), we conclude that $y-y_{0} \epsilon$ $\left\langle l, \eta\left(x, x_{0}\right)\right\rangle+D$.

Proposition 27. Let $A$ be a nonempty invex subset of $R^{m}$ and let $\eta: A \times A \rightarrow R^{m}$ be an open mapping such that it is affine in the first argument and $\eta(x, z)+\eta(z, x)=0, \forall x, z \in A$. Let $F$ : $A \rightarrow 2^{R^{n}}$ be strict pseudoinvex with respect to the same $\eta$ and $\partial F(x, y) \neq \emptyset$ for all $(x, y)$ with $y \in F(x)$ such that $t \partial F(x, y) \subseteq$ $\partial F(x, y)$ for all $t \in(0,1]$. If $\left(x_{0}, y_{0}\right) \in W \operatorname{Min}(F, A)$ and $\partial F$ is upper semicontinuous at $\left(x_{0}, y_{0}\right)$, then $\left(x_{0}, y_{0}\right) \in S_{W G V V L I}$.
Proof. Let $\left(x_{0}, y_{0}\right) \in W \operatorname{Min}(F, A)$. Consider any $x \in A$ and any sequence $\left\{\lambda_{n}\right\} \rightarrow 0$ with $\lambda_{n} \in(0,1]$. Since $A$ is invex,

$$
x_{n}=x_{0}+\lambda_{n} \eta\left(x, x_{0}\right) \in A .
$$

Since $\left(x_{0}, y_{0}\right) \in W \operatorname{Min}(F, A)$, we have

$$
\left(F\left(x_{n}\right)-y_{0}\right) \cap(-\operatorname{int} D)=\emptyset \text {. }
$$

That is,

$$
y_{0}-y_{n} \notin \operatorname{int} D, \quad \forall y_{n} \in F\left(x_{n}\right) .
$$

Since $D F\left(x_{0}, y_{0}\right)$ exists and $\eta\left(x, x_{0}\right) \in \operatorname{dom} D F\left(x_{0}, y_{0}\right)$, there exists $y_{n} \in F\left(x_{n}\right)$ such that $y_{n} \rightarrow y_{0}$ as $\lambda_{n} \rightarrow 0^{+}$. Moreover, $\partial F(x, y) \neq \emptyset$ at every $(x, y)$ with $y \in F(x)$ and $F$ is strict pseudoinvex with respect to $\eta$, so for all $l_{n} \in \partial F\left(x_{n}, y_{n}\right)$ we have

$$
\left\langle l_{n}, \eta\left(x_{0}, x_{n}\right)\right\rangle \notin \operatorname{int} D .
$$

Since $\eta$ is affine in the first argument and $\eta(x, z)+\eta(z, x)=0$, $\forall x, z \in A$, we have

$$
\left\langle l_{n}, \eta\left(x, x_{0}\right)\right\rangle \notin-\operatorname{int} D ;
$$

that is,

$$
\left\langle l_{n}, \eta\left(x, x_{0}\right)\right\rangle \in Y \backslash-\operatorname{int} D,
$$

where $Y \backslash-\operatorname{int} D$ is a closed cone. By assumptions and Remark 9, we get that $\partial F\left(x_{n}, y_{n}\right)$ is asymptotically compact closed convex set in $L\left(R^{n}, R^{m}\right)$. Furthermore, $\partial F$ being upper semicontinuous at $\left(x_{0}, y_{0}\right),\left(x_{n}, y_{n}\right) \rightarrow\left(x_{0}, y_{0}\right)$ and by Remark 10 and Lemma 25, consequently, $\left\{l_{n}\right\}$ asymptotically converges to some $l_{0} \in \partial F\left(x_{0}, y_{0}\right)$ with $l_{n} \in \partial F\left(x_{n}, y_{n}\right)$. If sequence $\left\{l_{n}\right\}$ is bounded, without loss of generality, we may assume that $l_{n} \rightarrow l_{0} \in \partial F\left(x_{0}, y_{0}\right)$; otherwise, if sequence $\left\{l_{n}\right\}$ with $\lim \left\|l_{n}\right\|=\infty$, we may also assume sequence $l_{n} /\left\|l_{n}\right\| \rightarrow$ $l_{0} \in \partial F\left(x_{0}, y_{0}\right)$; from these facts and $Y \backslash-$ int $D$ being a closed cone, it follows that

$$
\left\langle l_{0}, \eta\left(x, x_{0}\right)\right\rangle \in Y \backslash-\operatorname{int} D .
$$

Thus, $\forall x \in A, \exists l_{0} \in \partial F\left(x_{0}, y_{0}\right)$ such that

$$
\left\langle l_{0}, \eta\left(x, x_{0}\right)\right\rangle \notin-\operatorname{int} D \text {. }
$$

Hence, $\left(x_{0}, y_{0}\right) \in S_{\text {WGVVLI}}$.

Remark 28. Let $A=[0,5]$ and $\eta(x, y)=\alpha(x-y)$; then $A$ is an invex set and $\eta$ is affine in the first argument and continuous in the second argument, where $0<\alpha<1$.

Proposition 29. Let $A$ be a nonempty invex set of $R^{m}$ and $F$ : $A \rightarrow R^{n}$ subdifferentiable and D-preinvex with respect to the same $\eta: A \times A \rightarrow R^{m}$. If $\left(x_{0}, y_{0}\right)$ is a solution of WGVVLI, then it is also a weakly efficient solution of VOP.

Proof. Suppose that $\left(x_{0}, y_{0}\right)$ is a solution of WGVVLI, but not a generalized weakly efficient solution of VOP. Then there exist $\bar{x} \in A$ such that $F(\bar{x})-y_{0} \cap-\operatorname{int} D \neq \emptyset$. That is, $\exists \bar{y} \in F(\bar{x})$ such that $\bar{y}-y_{0} \in-$ int $D$. Since $F$ is subdifferentiable and $D$-preinvex with respect to the same $\eta$, by Proposition 26, for each $l_{0} \in \partial F\left(x_{0}, y_{0}\right)$, we have $\left\langle l_{0}, \eta\left(\bar{x}, x_{0}\right)\right\rangle \in \bar{y}-y_{0}-D \in-\operatorname{int} D-D \subset-$ int $D$, which contradicts $\left(x_{0}, y_{0}\right) \in S_{\text {WGVVLI}}$. 
Let $A$ be a nonempty convex subset of a vector space $X$. A mapping $F: A \rightarrow 2^{X}$ is said to be a KKM mapping if for each nonempty finite subset $K$ of $A$, $\operatorname{conv} K \subset F(K)$, where convK denotes the convex hull of $K$, and $F(K)=\bigcup\{F(x)$ : $x \in K\}$.

The following form of Fan-KKM lemma appeared in [22].

Lemma 30. Let $M$ be a convex subset of a Hausdorff topological vector space $X, \emptyset \neq A \subset M$. Let $\Gamma$ be set-valued maps such that the following conditions hold:

$\left(A_{1}\right) \Gamma$ is a KKM mapping on $A$;

$\left(A_{2}\right)$ for all $x \in A, \Gamma(x)$ is closed;

$\left(A_{3}\right)$ if there is a nonempty subset $A_{0}$ of $A$ such that the intersection $\bigcap_{x \in A_{0}} \Gamma(x)$ is compact and $A_{0}$ is contained in a compact convex subset of $M$,

then

$$
\bigcap_{x \in A} \Gamma(x) \neq \emptyset .
$$

Theorem 31. (i) Let $A$ be a nonempty convex subset of $R^{m}$ and let $\eta: A \times A \rightarrow R^{m}$ be an open mapping such that it is affine in the first argument and continuous in the second argument and $\eta(x, z)+\eta(z, x)=0, \forall x, z \in A$. Let $F: A \rightarrow 2^{R^{n}}$ be $D$-preinvex with respect to $\eta$ such that $\partial F(x, y)$ exists and $\partial F$ is upper semicontinuous at every $(x, y) \in \operatorname{gr} F$ with $x \in A$ and that $t \partial F(x, y) \subseteq \partial F(x, y)$ for all $t \in(0,1]$. If $\partial F$ is upper semicontinuous at each $(x, y) \in \operatorname{gr} F$ with $x \in A$.

(ii) Assume that there exists a nonempty compact convex set $B \subset A$ such that for each $z \in A \backslash B, \exists \tilde{x} \in B$, such that $\langle\partial F(z, w), \eta(\widetilde{x}, z)\rangle \subset-\operatorname{int} D$, where $w \in F(z)$.

Then, VOP has a generalized weakly efficient solution.

Proof. We define a multivalued map $\Gamma: A \rightarrow 2^{R^{n}}$ by

$$
\begin{array}{r}
\Gamma(x)=\{z \in A:\langle\partial F(z, w), \eta(x, z)\rangle \nsubseteq-\operatorname{int} D\} \\
=\left\{z \in A: \exists l_{z} \in \partial F(z, w)\right. \\
\text { such that } \left.\left\langle l_{z}, \eta(x, z)\right\rangle \in Y \backslash-\operatorname{int} D\right\} \\
\forall x \in A,
\end{array}
$$

where $w \in F(z)$ such that $\partial F(z, w) \neq \emptyset$. Then, $\forall x \in A, \Gamma(x)$ is closed in $R^{m}$. Indeed, let $\left\{z_{n}\right\}$ be a sequence in $\Gamma(x)$ such that $z_{n} \rightarrow z_{0} \in A$. Let $w_{0} \in F\left(z_{0}\right)$ such that $D F\left(z_{0}, w_{0}\right)$ exists. $F$ is epidifferentiable at $\left(z_{0}, w_{0}\right)$, so for each $z_{n}$ there exists $w_{n} \in$ $F\left(z_{n}\right)$ such that $w_{n} \rightarrow w_{0}$. Furthermore, $z_{n} \in \Gamma(x)$ implies there exist $l_{z n} \in \partial F\left(z_{n}, w_{n}\right)$ such that

$$
\left\langle l_{z n}, \eta\left(x, z_{n}\right)\right\rangle \in Y \backslash-\operatorname{int} D .
$$

Since $\partial F$ is upper semicontinuous at $\left(z_{0}, w_{0}\right),\left(z_{n}, w_{n}\right) \rightarrow$ $\left(z_{0}, w_{0}\right)$ and considering also Remark $10,\left\{l_{z_{n}}\right\}$ possesses an asymptotically convergent subnet. Without loss of generality, we may assume that the $l_{z_{n}} \rightarrow l_{z_{0}}\left(\right.$ or $l_{z_{n}} /\left\|l_{z_{n}}\right\| \rightarrow l_{z_{0}}$ ) and $l_{z_{0}} \in \partial F\left(z_{0}, w_{0}\right)$. Since $\eta$ is continuous in the second variable and $Y \backslash-$ int $D$ is a closed set, it is easy to check that $\left\langle l_{z_{0}}, \eta\left(x, z_{0}\right)\right\rangle \in Y \backslash-\operatorname{int} D$. So, $\Gamma(x)$ is a closed set. Next, we claim that $\Gamma$ is a $K K M$ mapping on $A$. Suppose that $\Gamma$ is not a KKM mapping; then there exist $\left\{x_{1}, x_{2}, \ldots, x_{n}\right\} \subset A$ and $t_{i} \geq 0$ with $\sum_{i=1}^{n} t_{i}=1$ such that $z=\sum_{i=1}^{n} t_{i} x_{i} \notin \bigcup_{i=1}^{n} \Gamma\left(x_{i}\right)$. Thus, $\exists l_{z} \in \partial F(z, w)$ such that, for any $i=1,2, \ldots, n$,

$$
\left\langle l_{z}, \eta\left(x_{i}, z\right)\right\rangle \in-\operatorname{int} D .
$$

Since int $D$ is a convex cone, we have

$$
\begin{aligned}
& \left\langle l_{z}, t_{1} \eta\left(x_{1}, z\right)\right\rangle+\left\langle l_{z}, t_{2} \eta\left(x_{2}, z\right)\right\rangle+\cdots+\left\langle l_{z}, t_{n} \eta\left(x_{n}, z\right)\right\rangle \\
& \quad \in-\operatorname{int} D .
\end{aligned}
$$

Since $\eta(\cdot, z)$ is affine, we get

$$
\left\langle l_{z}, \eta(z, z)\right\rangle \in-\operatorname{int} D .
$$

But from $\eta(x, z)+\eta(z, x)=0, \forall x, z \in A$, we have $\eta(z, z)=0$. Thus $\left\langle l_{z}, \eta(z, z)\right\rangle=0 \in-$ int $D$, which contradicts the fact $0 \notin$ - int $D$, so $\Gamma$ is a $K K M$ mapping. By condition (ii), $\bigcap_{x \in B} \Gamma(x)$ is a closed subset of a compact set $B$ and hence compact. Then by Lemma $30, \bigcap_{x \in A} \Gamma(x) \neq \emptyset$. That is, $\exists x_{0} \in A, y_{0} \in F\left(x_{0}\right)$ such that $l_{0} \in \partial F\left(x_{0}, y_{0}\right)$ and $\left\langle l_{0}, \eta\left(x, x_{0}\right)\right\rangle \notin-\operatorname{int} D, \forall x \in$ $A$. Thus, $\left(x_{0}, y_{0}\right) \in S_{\mathrm{WGVVLI}}$. From Proposition 29, we obtain $\left(x_{0}, y_{0}\right) \in W \operatorname{Min}(F, A)$. The proof is complete.

Remark 32. We note that our assumptions in Theorem 31 are totally different from those of Theorem 8 in [5] and Theorem 4.5 in [9] since we assume that $\partial F$ is upper semicontinuous and $t \partial F(x, y) \subseteq \partial F(x, y)$ for all $t \in(0,1]$ while correspondences $T$ and $\partial f$ in [5] and [9], respectively, were supposed to be pseudomonotone and $\Gamma(x)$ needs to be closed.

\section{Conflict of Interests}

The authors declare that there is no conflict of interests regarding the publication of this paper.

\section{Acknowledgment}

This work was supported by the National Science Foundation of China (nos. 61373174 and 11301407).

\section{References}

[1] F. Giannessi, "Theorems of the alternative, quadratic programs, and complementarity problems," in Variational Inequality and Complementarity Problems, W. R. Cottle and J. L. Lions, Eds., pp. 151-186, Wiley, Chichester, UK, 1980.

[2] G. Y. Chen, "Existence of solutions for a vector variational inequality: an extension of the Hartmann-Stampacchia theorem," Journal of Optimization Theory and Applications, vol. 74, no. 3, pp. 445-456, 1992.

[3] F. Giannessi, Ed., Vector Variational Inequalities and Vector Equilibria: Mathematical Theories, vol. 38, Kluwer Academic, Dordrecht, The Netherlands, 2000.

[4] A. H. Siddiqi, Q. H. Ansari, and R. Ahmad, "On vector variational-like inequalities," Indian Journal of Pure and Applied Mathematics, vol. 28, no. 8, pp. 1009-1016, 1997. 
[5] Q. H. Ansari, M. Rezaie, and J. Zafarani, "Generalized vector variational-like inequalities and vector optimization," Journal of Global Optimization, vol. 53, no. 2, pp. 271-284, 2012.

[6] Q. H. Ansari and A. H. Siddiqi, "A generalized vector variational-like inequality and optimization over an efficient set," in Functional Analysis with Current Applications in Science, Engineering, and Industry, pp. 177-191, Longman, Essex, UK, 1998.

[7] Q. H. Ansari and J. C. Yao, "On nondifferentiable and nonconvex vector optimization problems," Journal of Optimization Theory and Applications, vol. 106, no. 3, pp. 475-488, 2000.

[8] G. Ruiz-Garzón, R. A. Osuna-Gómez, and A. Rufián-Lizana, "Relationships between vector variational-like inequality and optimization problems," European Journal of Operational Research, vol. 157, no. 1, pp. 113-119, 2004.

[9] M. Rezaie and J. Zafarani, "Vector optimization and variationallike inequalities," Journal of Global Optimization, vol. 43, no. 1, pp. 47-66, 2009.

[10] J. Dutta, V. Vetrivel, and S. Nanda, "Semi-invex functions and their subdifferentials," Bulletin of the Australian Mathematical Society, vol. 56, no. 3, pp. 385-393, 1997.

[11] G. M. Lee, D. S. Kim, and H. Kuk, "Existence of solutions for vector optimization problems," Journal of Mathematical Analysis and Applications, vol. 220, no. 1, pp. 90-98, 1998.

[12] J. Li and G. Mastroeni, "Vector variational inequalities involving set-valued mappings via scalarization with applications to error bounds for gap functions," Journal of Optimization Theory and Applications, vol. 145, no. 2, pp. 355-372, 2010.

[13] M. Miholca, "On set-valued optimization problems and vector variational-like inequalities," Optimization Letters, vol. 8, no. 2, pp. 463-476, 2014.

[14] S. K. Mishra, S. Y. Wang, and K. K. Lai, "Gap function for set-valued vector variational-like inequalities," Journal of Optimization Theory and Applications, vol. 138, no. 1, pp. 77-84, 2008.

[15] X. Q. Yang and J. C. Yao, "Gap functions and existence of solutions to set-valued vector variational inequalities," Journal of Optimization Theory and Applications, vol. 115, no. 2, pp. 407$417,2002$.

[16] J. Zeng and S. J. Li, "On vector variational-like inequalities and set-valued optimization problems," Optimization Letters, vol. 5, no. 1, pp. 55-69, 2011.

[17] J. Jahn and R. Raüh, "Contingent epiderivatives and set-valued optimization," Mathematical Methods of Operations Research, vol. 46, no. 2, pp. 193-211, 1997.

[18] J. Jahn and A. A. Khan, "The existence of contingent epiderivatives for set-valued maps," Applied Mathematics Letters, vol. 16, no. 8, pp. 1179-1185, 2003.

[19] J. Baier and J. Jahn, "On subdifferentials of set-valued maps," Journal of Optimization Theory and Applications, vol. 100, no. 1, pp. 233-240, 1999.

[20] P. Q. Khanh and N. D. Tuan, "First and second-order approximations as derivatives of mappings in optimality conditions for nonsmooth vector optimization," Applied Mathematics and Optimization, vol. 58, no. 2, pp. 147-166, 2008.

[21] A. P. Farajzadeh, A. Amini-Harandi, and K. R. Kazmi, "Existence of solutions to generalized vector variational-like inequalities," Journal of Optimization Theory and Applications, vol. 146, no. 1, pp. 95-104, 2010.

[22] K. Fan, "Some properties of convex sets related to fixed point theorems," Mathematische Annalen, vol. 266, no. 4, pp. 519-537, 1984. 


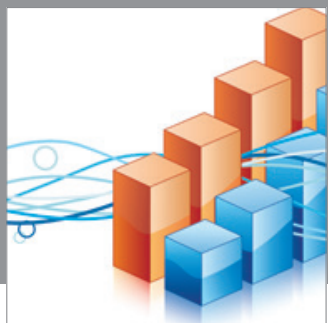

Advances in

Operations Research

mansans

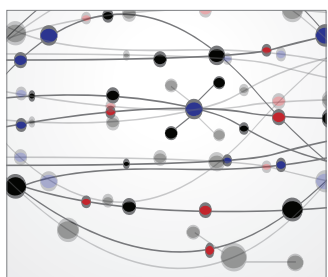

The Scientific World Journal
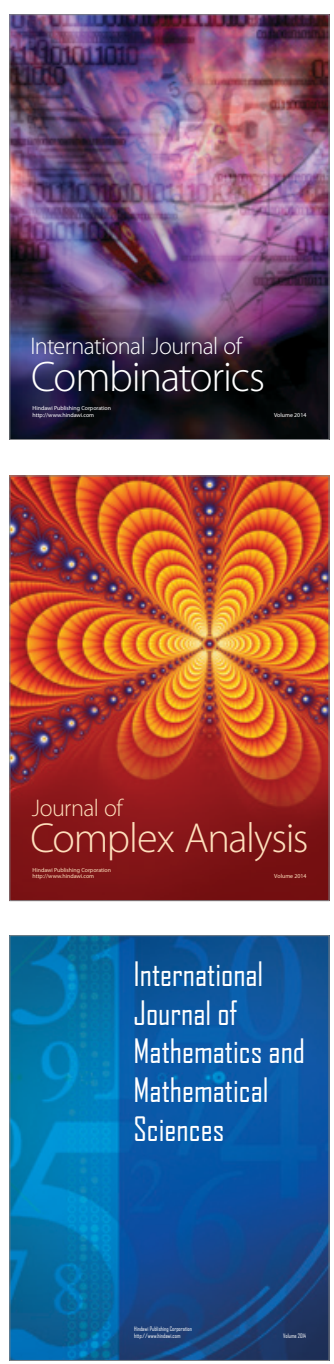
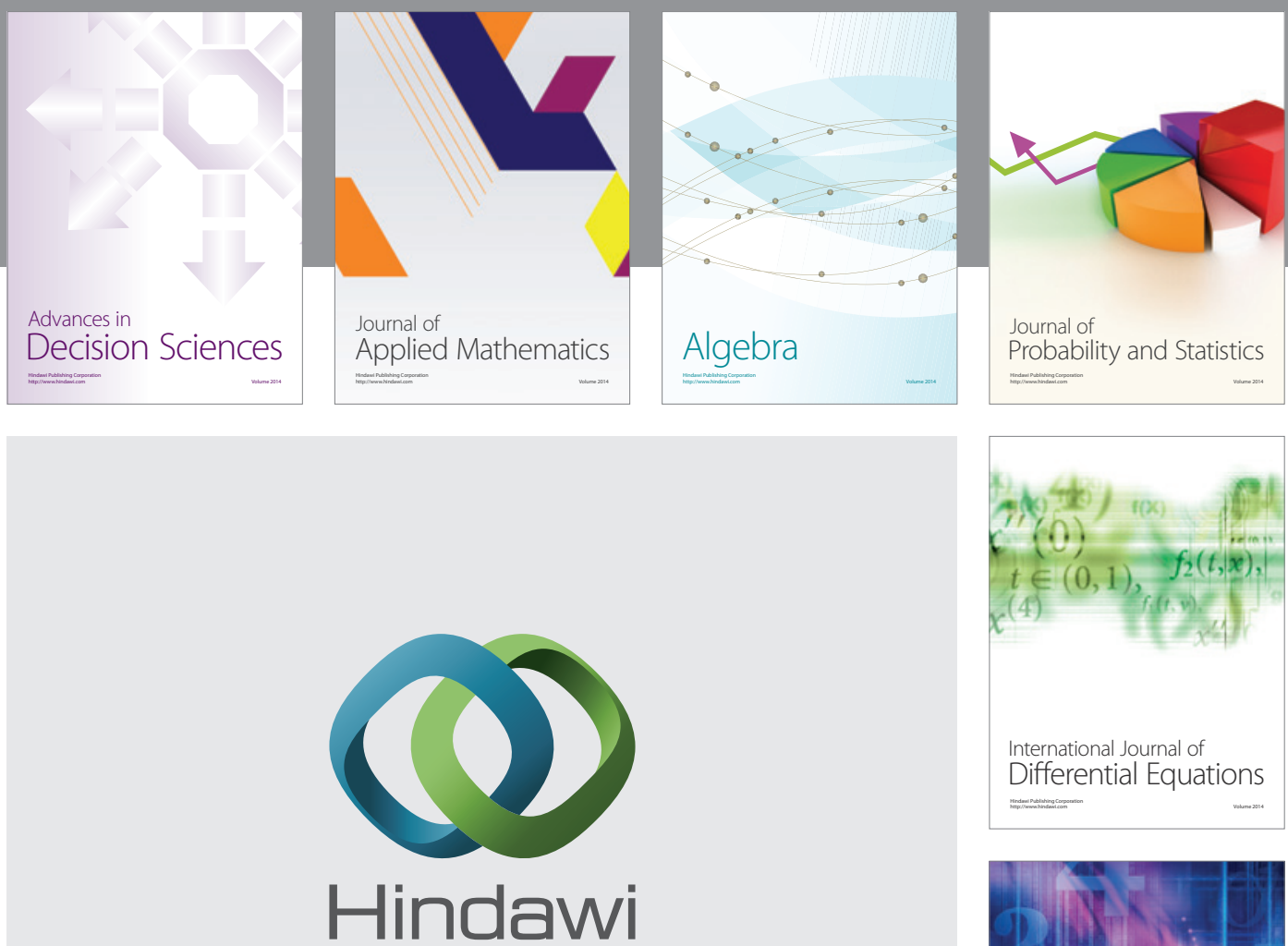

Submit your manuscripts at http://www.hindawi.com
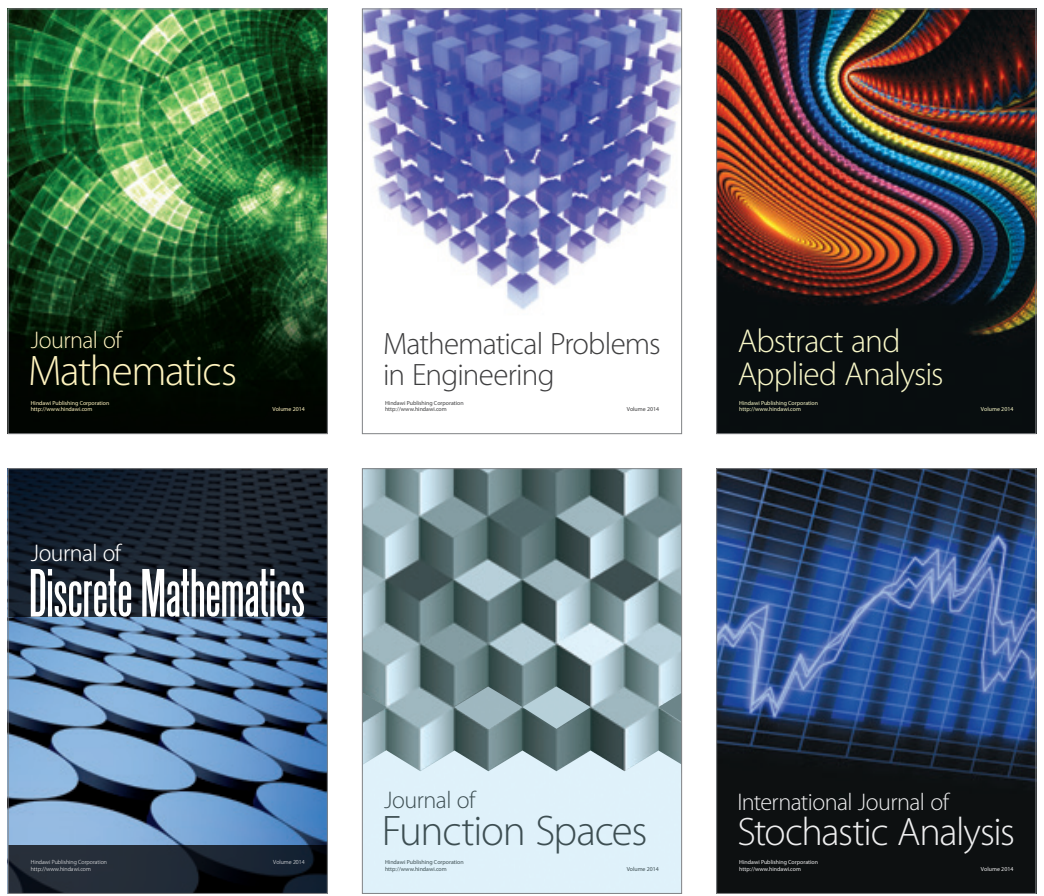

Journal of

Function Spaces

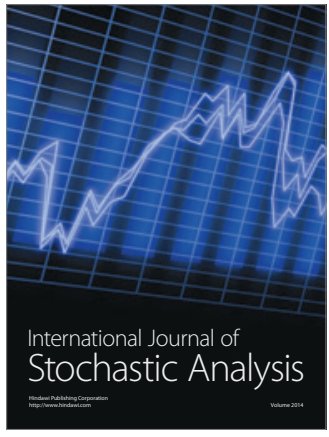

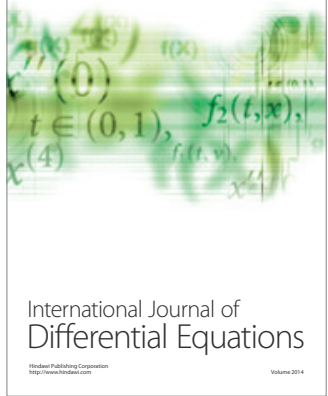
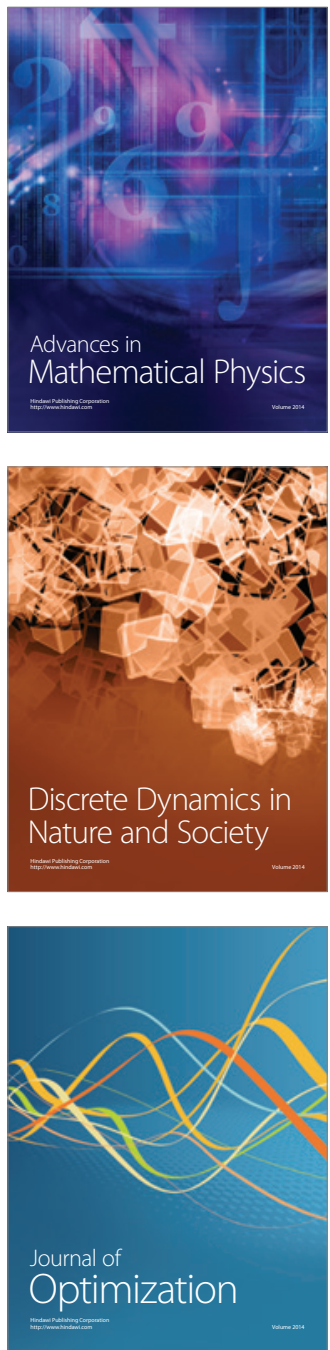